Vietnam Journal of Mechanics, VAST, Vol.39, No.3 (2017), pp. 245-257

DOI:10.15625/0866-7136/9692

\title{
NONLINEAR VIBRATION OF FUNCTIONALLY GRADED MATERIAL SANDWICH DOUBLY CURVED SHALLOW SHELLS REINFORCED BY FGM STIFFENERS. PART 1: GOVERNING EQUATIONS
}

\author{
Dang Thuy Dong ${ }^{1, *}$, Dao Van Dung ${ }^{2}$ \\ ${ }^{1}$ University of Transport Technology, Hanoi, Vietnam \\ ${ }^{2}$ VNU University of Science, Hanoi, Vietnam \\ *E-mail: dongdt@utt.edu.vn \\ Received April 19, 2017
}

\begin{abstract}
Nonlinear vibration of FGM sandwich doubly curved shallow shells reinforced by FGM stiffeners subjected to mechanical and thermal loading are investigated based on the first-order shear deformation theory (FSDT) with von Karman type nonlinearity, taking into account initial geometrical imperfection and smeared stiffener technique. Four material models of the FGM sandwich shells are presented. Explicit expressions for natural frequencies, nonlinear frequency-amplitude relation, and time-deflection curves of the FGM sandwich shallow shells are derived using Galerkin method.
\end{abstract}

Keywords: Nonlinear vibration, FGM sandwich shallow shell, stiffener.

\section{INTRODUCTION}

Functionally graded material (FGM) sandwich structures with advantage properties as: high stiffness, high structural efficiency, strength, ... have attracted attention of many researchers. For FGM sandwich plates, Xia and Shen [1] considered the smalland large-amplitude vibrations of compressively and thermally post-buckled sandwich plates with FGM face sheets in thermal environments based on a high-order shear deformation plate theory (HSDT) and a general von Karman-type equation that includes a thermal effect. Improved high-order sandwich plate theory is used to analyze the free vibration of sandwich plates with functionally graded face sheets in various thermal environments by Khalili and Mohammadi [2]. Pandey and Pradyumna [3] investigated the free vibration of sandwich plates made of FGM in the thermal environment by using a layerwise theory.

For FGM sandwich shells, by employing the generalized differential quadrature method, Aragh and Yas [4] studied the effect of continuously grading fiber orientation

(C) 2017 Vietnam Academy of Science and Technology 
face sheets on free vibration of sandwich panels with functionally graded core using generalized power-law distribution. Dey and Ramachandra [5] investigated the buckling and post-buckling responses of cylindrical sandwich panels subjected to non-uniform inplane loadings by analytical method. The dynamic instability of three-layered cylindrical shells containing a functionally graded interlayer subjected to static and time dependent periodic axial compressive loads were presented by Sofiyev and Kuruoglu [6]. Dung and Dong $[7,8]$ analyzed the nonlinear thermo-mechanical stability of FGM sandwich doubly curved shallow shells resting on elastic foundations based on FSDT and HSDT.

Recently, the nonlinear dynamic response and vibration of imperfect eccentrically stiffened FGM doubly curved shallow shells subjected to mechanical and thermal loads on elastic foundation were studied by Bich et al [9] using FSDT, and Duc and Quan [10] based on the classical shell theory (CST). In their papers, a simple power-law distribution (P-FGM) was used and the shell was reinforced by homogeneous stiffeners. The thermal elements of stiffeners in expression of force and moment resultants weren't considered. The present paper extends the previous work [7] to investigate nonlinear vibration of FGM sandwich shallow shells with some contributions: FGM sandwich doubly curved shallow shells reinforced by FGM stiffeners; the thermal elements of stiffeners in expression of force and moment resultants are considered; analytical expression for Young's modulus, thermal expansion coefficient and mass density of shells and stiffeners are presented for four material models of FGM.

\section{ECCENTRICALLY STIFFENED FGM SANDWICH SHALLOW SHELL (ES-FGM SANDWICH SHALLOW SHELL)}

Consider a sandwich doubly curved shallow shell which has the principal radii of curvature $R_{x}$ and $R_{y}$; the in-plane edges $a$ and $b$; the thickness $h$. The shell is reinforced by closely spaced eccentrically longitudinal and transversal FGM stiffeners and rested on a Pasternak elastic foundation as shown in Fig. 1.
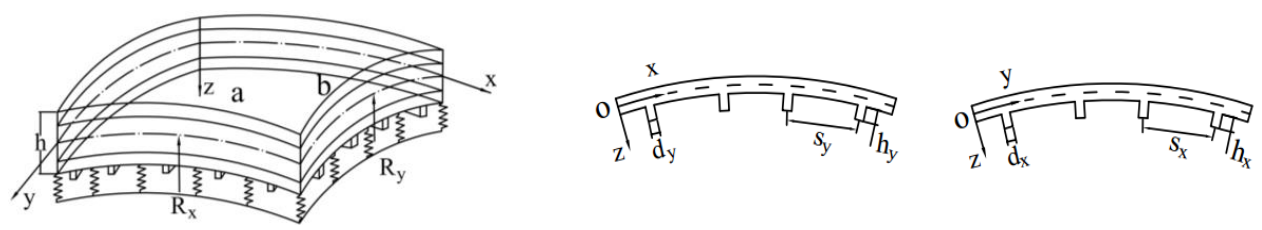

Fig. 1. Geometry and coordinate system of a sandwich doubly curved shallow shell reinforced by FGM stiffeners on elastic foundations

Assume that shell consists of three layers, the top face sheet, core layer, and bottom face sheet with thickness $h_{t}, h_{c}$ and $h_{b}$, respectively. These layers are made of FGMs or homogeneous materials.

\subsection{Sandwich shallow shell of FGM-homogeneous core-FGM model}

Four material models of FGM sandwich shallow shells are shown in Figs. 2(a) and $2(b)$. In these cases, the effective properties of the shell are varied in the thickness 


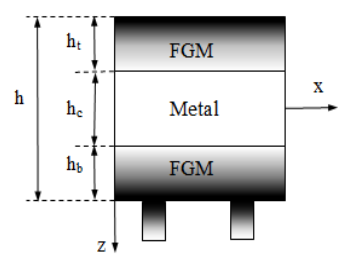

(a) Case $1 \mathrm{~A}$

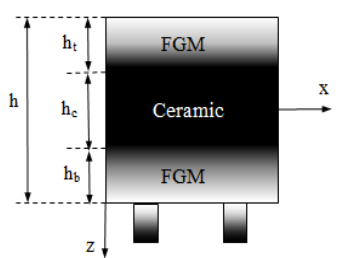

(b) Case 1B

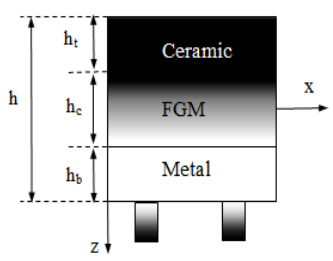

(c) Case $2 \mathrm{~A}$

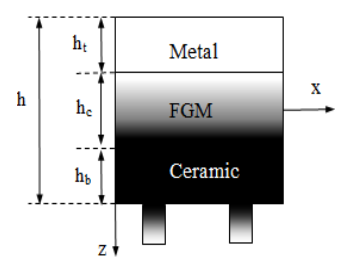

(d) Case 2B

Fig. 2. Four material models of the FGM sandwich shells with FGM stiffeners

direction according to general sigmoid law distribution as

$$
\left[E_{s h}, \alpha_{s h}, \rho_{s h}\right]=\left\{\begin{array}{l}
{\left[E_{i}, \alpha_{i}, \rho_{i}\right]+\left[E_{k l}, \alpha_{k l}, \rho_{k l}\right]\left(\frac{2 z+h}{2 h_{t}}\right)^{k_{t}} \text { for }-\frac{h}{2} \leq z \leq-\frac{h}{2}+h_{t},} \\
{\left[E_{j}, \alpha_{j}, \rho_{j}\right] \quad \text { for }-\frac{h}{2}+h_{t} \leq z \leq \frac{h}{2}-h_{b}} \\
{\left[E_{i}, \alpha_{i}, \rho_{i}\right]+\left[E_{k l}, \alpha_{k l}, \rho_{k l}\right]\left(\frac{-2 z+h}{2 h_{b}}\right)^{k_{b}} \text { for } \frac{h}{2}-h_{b} \leq z \leq \frac{h}{2}}
\end{array}\right.
$$

where $i=c, j=m, k l=m c$ for case $1 \mathrm{~A}$ and $i=m, j=c, k l=c m$ for case $1 \mathrm{~B}$ and

$$
\begin{aligned}
& E_{m c}(T)=E_{m}(T)-E_{c}(T), \alpha_{m c}(T)=\alpha_{m}(T)-\alpha_{c}(T), \rho_{m c}(T)=\rho_{m}(T)-\rho_{c}(T), \\
& E_{c m}(T)=E_{c}(T)-E_{m}(T), \alpha_{c m}(T)=\alpha_{c}(T)-\alpha_{m}(T), \rho_{c m}(T)=\rho_{c}(T)-\rho_{m}(T),
\end{aligned}
$$

$k_{t}$ and $k_{b}$ are volume fraction indices of top FGM face sheet and bottom FGM face sheet, and the subscripts $m, c$, sh denote the metal, ceramic, shell, respectively.

It is worth noting that, from these laws when $h_{t}=h_{b}=h / 2$ and $k_{t}=k_{b}$, we obtain known-well symmetric S-FGM laws.

\subsection{Sandwich shallow shell of homogeneous-FGM core-homogeneous model}

Figs. 2(c) and 2(d) show sandwich shells of homogeneous-FGM core-homogeneous model. The effective properties of sandwich shell of these models are expressed as follow

$$
\left[E_{s h}, \alpha_{s h}, \rho_{s h}\right]= \begin{cases}{\left[E_{c}, \alpha_{c}, \rho_{c}\right]} & \text { for }-\frac{h}{2} \leq z \leq-\frac{h}{2}+h_{t}, \\ {\left[E_{c}, \alpha_{c}, \rho_{c}\right]+\left[E_{m c}, \alpha_{m c}, \rho_{m c}\right]\left(\frac{2 z+h-2 h_{t}}{2 h_{c}}\right)^{k}} & \text { for }-\frac{h}{2}+h_{t} \leq z \leq \frac{h}{2}-h_{b} \\ {\left[E_{m}, \alpha_{m}, \rho_{m}\right]} & \text { for } \frac{h}{2}-h_{b} \leq z \leq \frac{h}{2} .\end{cases}
$$

where $i=c, j=m, k l=m c$ for case $1 \mathrm{~A}, i=m, j=c, k l=c m$ for case 1B:

The law (2) generalizes the power law distribution. It can be seen that when $h_{t}=$ $h_{b}=0$, this laws return to the known-well P-FGM laws. 


\subsection{FGM stiffeners}

In order to guarantee the continuity between shell and stiffeners, effective properties of stiffeners are given by

$$
\begin{aligned}
& {\left[E_{s x}, \alpha_{s x}, \rho_{s x}\right]=\left[E_{i}, \alpha_{i}, \rho_{i}\right]+\left[E_{k l}, \alpha_{k l}, \rho_{k l}\right]\left(\frac{2 z-h}{2 h_{x}}\right)^{k_{2}}, h / 2 \leq z \leq h / 2+h_{x},} \\
& {\left[E_{s y}, \alpha_{s y}, \rho_{s y}\right]=\left[E_{i}, \alpha_{i}, \rho_{i}\right]+\left[E_{k l}, \alpha_{k l}, \rho_{k l}\right]\left(\frac{2 z-h}{2 h_{y}}\right)^{k_{3}}, h / 2 \leq z \leq h / 2+h_{y} .}
\end{aligned}
$$

For case 1A and case 2B: $i=c, k l=m c$ and for case 1B and case 2A: $i=m, k l=c m$.

In Eq. (3), $k_{2}, k_{3}$ are the volume fraction indices and $h_{x}, h_{y}$ are the thickness of longitudinal and transversal stiffeners, respectively; the subscripts $s$ denote the stiffener.

\section{GOVERNING EQUATIONS}

The strain components across the shell thickness at a distance $z$ from the middle surface are $[7,9]$

$$
\left(\begin{array}{c}
\varepsilon_{x} \\
\varepsilon_{y} \\
\gamma_{x y}
\end{array}\right)=\left(\begin{array}{c}
u_{, x}-w / R_{x}+\frac{1}{2} w_{, x}^{2}+w_{, x} w_{, x}^{*} \\
v_{, y}-w / R_{y}+\frac{1}{2} w_{, y}^{2}+w_{, y} w_{, y}^{*} \\
u_{, y}+v_{, x}+w_{, x} w_{, y}+w_{, x} w_{, y}^{*}+w_{, y} w_{, x}^{*}
\end{array}\right)+z\left(\begin{array}{c}
\psi_{x, x} \\
\psi_{y, y} \\
\psi_{x, y}+\psi_{y, x}
\end{array}\right),\left(\begin{array}{c}
\gamma_{x z} \\
\gamma_{y z}
\end{array}\right)=\left(\begin{array}{c}
\psi_{x}+w, x \\
\psi_{y}+w_{, y}
\end{array}\right),
$$

where $\varepsilon_{x}, \varepsilon_{y}$ are the normal strains, $\gamma_{x y}$ is the in-plane shear strain, and $\gamma_{x z}, \gamma_{y z}$ are the transverse shear deformations; whereas $u, v$ and $w$ are the displacement components along the $x, y, z$ directions, respectively. $\psi_{x}$ and $\psi_{y}$ are the rotations of normal to the middle surface with respect to $y$ and $x$ axes, respectively. Also, $w^{*}=w^{*}(x, y)$ is the initial imperfection and assumed very small in comparison with the shell thickness.

Hooke's law for FGM sandwich shallow shell and FGM stiffeners taking into account the temperature is defined as

For shells

$$
\begin{aligned}
& \left(\sigma_{x}^{s h}, \sigma_{y}^{s h}\right)=\frac{E_{s h}(z, T)}{1-v^{2}}\left[\left(\varepsilon_{x}, \varepsilon_{y}\right)+v\left(\varepsilon_{y}, \varepsilon_{x}\right)-(1+v) \alpha_{s h}(z, T) \Delta T(1,1)\right], \\
& \left(\sigma_{x y}^{s h}, \sigma_{x z}^{s h}, \sigma_{y z}^{s h}\right)=\frac{E_{s h}(z, T)}{2(1+v)}\left(\gamma_{x y}, \gamma_{x z}, \gamma_{y z}\right) .
\end{aligned}
$$

For stiffeners

$$
\begin{aligned}
& \sigma_{x}^{s}=E_{s x}(z, T) \varepsilon_{x}-E_{s x}(z, T) \alpha_{s x}(z, T) \Delta T, \sigma_{y}^{s}=E_{s y}(z, T) \varepsilon_{y}-E_{s y}(z, T) \alpha_{s y}(z, T) \Delta T, \\
& \sigma_{x z}^{s}=G_{s x} \gamma_{x z}, \sigma_{y z}^{s}=G_{s y} \gamma_{y z} .
\end{aligned}
$$

Using the Lekhnitskii smeared stiffener technique and integrating the stress-strain equations through the thickness of the shell, we obtain the expression of the force and moment resultants of a ES-FGM sandwich doubly curved shallow shell [7] 


$$
\begin{aligned}
& N_{x}=\left(H_{11}+\frac{E_{1 x} d_{x}}{s_{x}}\right) \varepsilon_{x}^{0}+H_{12} \varepsilon_{y}^{0}+\left(G_{11}+\frac{E_{2 x} d_{x}}{s_{x}}\right) \psi_{x, x}+G_{12} \psi_{y, y}-\frac{1}{1-v} \varphi_{1}^{s h}-\frac{d_{x}}{s_{x}} \varphi_{1}^{s x}, \\
& N_{y}=H_{12} \varepsilon_{x}^{0}+\left(H_{22}+\frac{E_{1 y} d_{y}}{s_{y}}\right) \varepsilon_{y}^{0}+G_{12} \psi_{x, x}+\left(G_{22}+\frac{E_{2 y} d_{y}}{s_{y}}\right) \psi_{y, y}-\frac{1}{1-v} \varphi_{1}^{s h}-\frac{d_{y}}{s_{y}} \varphi_{1}^{s y}, \\
& N_{x y}=H_{66} \gamma_{x y}^{0}+G_{66}\left(\psi_{x, y}+\psi_{y, x}\right), \\
& M_{x}=\left(G_{11}+\frac{E_{2 x} d_{x}}{s_{x}}\right) \varepsilon_{x}^{0}+G_{12} \varepsilon_{y}^{0}+\left(L_{11}+\frac{E_{3 x} d_{x}}{s_{x}}\right) \psi_{x, x}+L_{12} \psi_{y, y}-\frac{1}{1-v} \varphi_{2}^{s h}-\frac{d_{x}}{s_{x}} \varphi_{2}^{s x}, \\
& M_{y}=G_{12} \varepsilon_{x}^{0}+\left(G_{22}+\frac{E_{2 y} d_{y}}{s_{y}}\right) \varepsilon_{y}^{0}+L_{12} \psi_{x, x}+\left(L_{22}+\frac{E_{3 y} d_{y}}{s_{y}}\right) \psi_{y, y}-\frac{1}{1-v} \varphi_{2}^{s h}-\frac{d_{y}}{s_{y}} \varphi_{2}^{s y}, \\
& M_{x y}=G_{66} \gamma_{x y}^{0}+L_{66}\left(\psi_{x, y}+\psi_{y, x}\right) .
\end{aligned}
$$

The transverse force resultants are

$$
Q_{x}=H_{44} w_{, x}+H_{44} \psi_{x}, \quad Q_{y}=H_{55} w_{, y}+H_{55} \psi_{y},
$$

where $s_{x}$ and $s_{y}$ are the spacing of the longitudinal and transversal stiffeners, $h_{x}, d_{x}$ and $h_{y}, d_{y}$ are thickness and width for longitudinal stiffeners ( $x$-direction) and transversal stiffeners (y-direction), respectively. Expressions of $H_{i j}, G_{i j}, L_{i j}$ and $E_{i}, E_{i x}, E_{i y}(i=1 \div 3)$ and $\varphi_{i}^{s h}, \varphi_{i}^{s x}, \varphi_{i}^{s y}(i=1,2)$ are given in Appendix I.

In systems of Eqs. (7)-(9), FGM stiffeners and thermal elements are taken into account in calculations for sandwich shallow shell. This is one of important contributions of the present study.

The stress function $f=f(x, y)$ is introduced as

$$
N_{x}=f_{, y y}, \quad N_{y}=f_{, x x}, \quad N_{x y}=-f_{, x y} .
$$

The nonlinear motion equations of an imperfect FGM sandwich shallow shell on elastic foundations with damping force based on FSDT are of the form [7,9]

$$
\begin{gathered}
H_{44} w_{, x x}+H_{55} w_{, y y}+H_{44} \psi_{x, x}+H_{55} \psi_{y, y}+f_{, y y}\left(w_{, x x}+w_{, x x}^{*}\right)-2 f_{, x y}\left(w_{, x y}+w_{, x y}^{*}\right) \\
+f_{, x x}\left(w_{, y y}+w_{, y y}^{*}\right)+\frac{f_{, y y}}{R_{x}}+\frac{f_{, x x}}{R_{y}}+q-K_{1} w+K_{2}\left(w_{, x x}+w_{, y y}\right)=I_{1} w_{, t t}+2 \varepsilon I_{1} w_{, t}, \\
G_{21}^{*} f_{, x x x}+\left(G_{11}^{*}-G_{66}^{*}\right) f_{, x y y}+L_{11}^{*} \psi_{x, x x}+L_{66}^{*} \psi_{x, y y}+\left(L_{12}^{*}+L_{66}^{*}\right) \psi_{y, x y}-H_{44}\left(w_{, x}+\psi_{x}\right)=\bar{I}_{3} \psi_{x, t t}, \\
G_{12}^{*} f_{, y y y}+\left(G_{22}^{*}-G_{66}^{*}\right) f_{, x x y}+\left(L_{21}^{*}+L_{66}^{*}\right) \psi_{x, x y}+L_{22}^{*} \psi_{y, y y}+L_{66}^{*} \psi_{y, x x}-H_{55}\left(w_{, y}+\psi_{y}\right)=\bar{I}_{3} \psi_{y}, t t,
\end{gathered}
$$

where $\varepsilon$ is damping coefficient and $\bar{I}_{3}=-\frac{I_{2}^{2}}{I_{1}}+I_{3}$, in which

$$
\left(I_{1}, I_{2}, I_{3}\right)=\int_{-h / 2}^{h / 2} \rho(z, T)\left(1, z, z^{2}\right) d z+\frac{b_{x}}{d_{x}} \int_{h / 2}^{h / 2+h_{x}} \rho_{s x}(z, T)\left(1, z, z^{2}\right) d z+\frac{b_{y}}{d_{y}} \int_{h / 2}^{h / 2+h_{y}} \rho_{s y}(z, T)\left(1, z, z^{2}\right) d z .
$$


The deformation compatibility equation is given by [7]

$$
\begin{aligned}
& H_{11}^{*} f_{, x x x x}+\left(H_{66}^{*}-2 H_{12}^{*}\right) f_{, x x y y}+H_{22}^{*} f_{, y y y y}-G_{21}^{*} \psi_{x, x x x}-G_{12}^{*} \psi_{y, y y y}+\left(G_{66}^{*}-G_{11}^{*}\right) \psi_{x, x y y} \\
& +\left(G_{66}^{*}-G_{22}^{*}\right) \psi_{y, x x y}-\left(w_{, x y}^{2}-w_{, x x} w_{, y y}-w_{, x x} w_{, y y}^{*}+2 w_{, x y} w_{, x y}^{*}-w_{, x x}^{*} w_{, y y}-\frac{w_{, y y}}{R_{x}}-\frac{w_{, x x}}{R_{y}}\right)=0 .
\end{aligned}
$$

where $H_{i j}^{*}, G_{i j}^{*}, L_{i j}^{*}$ are determined in Appendix II.

Eqs. (11)-(14) including four dependent unknown functions $w, \psi_{x}, \psi_{y}$ and $f$ are used to investigate the nonlinear vibration of ES-FGM sandwich doubly curved shallow shell on elastic foundations.

\section{NONLINEAR VIBRATION ANALYSIS}

Two boundary conditions are considered in this study as follows.

Case 1. The four edges of the shell are simply supported and freely movable (FM), so that the boundary conditions are

$$
\begin{array}{ll}
w=M_{x}=N_{x y}=\psi_{y}=0, & N_{x}=N_{x 0}, \text { on } x=0, a, \\
w=M_{y}=N_{x y}=\psi_{x}=0, & N_{y}=N_{y 0}, \text { on } y=0, b .
\end{array}
$$

Case 2. The four edges of the shell are simply supported and immovable (IM), so that the boundary conditions are

$$
\begin{array}{ll}
w=u=\psi_{y}=M_{x}=0, & N_{x}=N_{x 0}, \text { on } x=0, a, \\
w=v=\psi_{x}=M_{y}=0, & N_{y}=N_{y 0}, \text { on } y=0, b .
\end{array}
$$

where $N_{x 0}$ and $N_{y 0}$ are prebuckling force resultants in directions $x$ and $y$, respectively.

The approximate solutions of Eqs. (11)-(14) and satisfying the boundary conditions (15), (16) are assumed in the form

$$
\left(w, w^{*}\right)=(W, \xi h) \sin \alpha x \sin \beta y, \quad \psi_{x}=\Psi_{x} \cos \alpha x \sin \beta y, \quad \psi_{y}=\Psi_{y} \sin \alpha x \cos \beta y,
$$

where $\alpha=m \pi / a, \beta=n \pi / b$ and $m, n$ are numbers of half waves in $x$ and $y$ directions, respectively, and $W, \Psi_{x}, \Psi_{y}$ are amplitudes of deflection and slope rotations, respectively. The coefficient $\xi \in[-0.5,0.5]$ is an imperfection size of the shell.

Substituting the expressions (17) into Eq. (14) and solving the resulting equation, the expression of the stress function $f$ is obtained as

$$
f=f_{1} \cos 2 \alpha x+f_{2} \cos 2 \beta y++f_{3} \sin \alpha x \sin \beta y+\frac{1}{2} N_{x 0} y^{2}+\frac{1}{2} N_{y 0} x^{2},
$$

in which

$$
\begin{aligned}
f_{1}= & \frac{\beta^{2}}{32 H_{11}^{*} \alpha^{2}} W(W+2 \xi h), \quad f_{2}=\frac{\alpha^{2}}{32 H_{22}^{*} \beta^{2}} W(W+2 \xi h), \\
f_{3}= & \frac{1}{\left[H_{11}^{*} \alpha^{4}+H_{22}^{*} \beta^{4}+\left(H_{66}^{*}-2 H_{12}^{*}\right) \alpha^{2} \beta^{2}\right]}\left(\frac{\beta^{2}}{R_{x}}+\frac{\alpha^{2}}{R_{y}}\right) W+ \\
& +\frac{\left[G_{21}^{*} \alpha^{3} \Psi_{x}+G_{12}^{*} \beta^{3} \Psi_{y}+\left(G_{11}^{*}-G_{66}^{*}\right) \alpha \beta^{2} \Psi_{x}+\left(G_{22}^{*}-G_{66}^{*}\right) \alpha^{2} \beta \Psi_{y}\right]}{\left[H_{11}^{*} \alpha^{4}+H_{22}^{*} \beta^{4}+\left(H_{66}^{*}-2 H_{12}^{*}\right) \alpha^{2} \beta^{2}\right]} .
\end{aligned}
$$


Substituting the expressions of $f, w, w^{*}$ into the Eqs. (11)-(13) and applying Galerkin method to the resulting equations yields

$$
\begin{gathered}
a_{11} W+a_{12} \Psi_{x}+a_{13} \Psi_{y}+a_{14}(W+\xi h) \Psi_{x}+a_{15}(W+\xi h) \Psi_{y} \\
+b_{1} W(W+\xi h)+b_{2} W(W+2 \xi h)+b_{3} W(W+\xi h)(W+2 \xi h) \\
+b_{4} q-\left(N_{x 0} \alpha_{m}^{2}+N_{y 0} \beta_{n}^{2}\right)(W+\xi h)+b_{4}\left(\frac{N_{x 0}}{R_{x}}+\frac{N_{y 0}}{R_{y}}\right)=I_{1} \ddot{W}+2 \varepsilon I_{1} \dot{W}, \\
a_{21} W+a_{22} \Psi_{x}+a_{23} \Psi_{y}+b_{5} W(W+2 \xi h)=\bar{I}_{3} \ddot{\Psi}_{x}, \\
a_{31} W+a_{32} \Psi_{x}+a_{33} \Psi_{y}+b_{6} W(W+2 \xi h)=\bar{I}_{3} \ddot{\Psi}_{y},
\end{gathered}
$$

where $\delta_{m}=(-1)^{m}-1, \delta_{n}=(-1)^{n}-1$ and $a_{i j}, b_{i}$ are given in Appendix III.

The system of Eqs. (20)-(22) is used to analyze the nonlinear vibration of the imperfect stiffened FGM sandwich shallow shells on elastic foundations under mechanical and thermo-mechanical loads.

\subsection{Nonlinear vibration analysis of the shells only under external pressure}

Consider an imperfect ES-FGM sandwich shallow shell with all freely-movable edges (Case 1) subjected to uniformly distributed transverse load $q=Q \sin \Omega t$. In this case, Eqs. (20)-(22) become

$$
\begin{gathered}
a_{11} W+a_{12} \Psi_{x}+a_{13} \Psi_{y}+a_{14}(W+\xi h) \Psi_{x}+a_{15}(W+\xi h) \Psi_{y} \\
+b_{1} W(W+\xi h)+b_{2} W(W+2 \xi h)+b_{3} W(W+\xi h)(W+2 \xi h) \\
+b_{4} Q \sin \Omega t-\left(N_{x 0} \alpha_{m}^{2}+N_{y 0} \beta_{n}^{2}\right)(W+\xi h)+b_{4}\left(\frac{N_{x 0}}{R_{x}}+\frac{N_{y 0}}{R_{y}}\right)=I_{1} \ddot{W}+2 \varepsilon I_{1} \dot{W}, \\
a_{21} W+a_{22} \Psi_{x}+a_{23} \Psi_{y}+b_{5} W(W+2 \xi h)=\bar{I}_{3} \ddot{\Psi}_{x}, \\
a_{31} W+a_{32} \Psi_{x}+a_{33} \Psi_{y}+b_{6} W(W+2 \xi h)=\bar{I}_{3} \ddot{\Psi}_{y} .
\end{gathered}
$$

From these equations, dynamic responses of ES-FGM sandwich shallow shells are obtained by using the four-order Runge-Kutta method. The following will present some cases which we can obtain explicit expressions to determine the natural frequencies, nonlinear frequency-amplitude relation of the sandwich shell under the mechanical load.

\subsubsection{Natural frequencies}

Taking linear part of the system Eqs. (23)-(25) and putting $q=0$, the natural frequencies of the perfect shells are determined by solving the following determinant

$$
\left|\begin{array}{ccc}
a_{11}+I_{1} \omega^{2} & a_{12} & a_{13} \\
a_{21} & a_{22}+\bar{I}_{3} \omega^{2} & a_{23} \\
a_{31} & a_{32} & a_{33}+\bar{I}_{3} \omega^{2}
\end{array}\right|=0 .
$$

If $\ddot{\Psi}_{x}$ and $\ddot{\Psi}_{y}$ are assumed to be very small and can be ignored, by solving $\Psi_{x}, \Psi_{y}$ from Eqs. (24), (25) and substituting them into Eq. (23) gives

$$
\begin{aligned}
I_{1} \ddot{W}+2 \varepsilon I_{1} \dot{W}-a_{1} W & -a_{2} W(W+2 \xi h)-a_{3} W(W+\xi h) \\
& -a_{4} W(W+\xi h)(W+2 \xi h)=b_{4} Q \sin \Omega t,
\end{aligned}
$$


where $a_{i}(i=1 \div 4)$ are given in Appendix IV. is given

From Eq. (27), the explicit expression of the natural frequency of perfect shell

$$
\omega_{m n}=\sqrt{-\frac{a_{1}}{I_{1}}}
$$

\subsubsection{Frequency-amplitude relation}

Consider the perfect shell and the vibration is nonlinear forced vibration, Eq. (27) is of the form

$$
\ddot{W}+2 \varepsilon \dot{W}+\omega_{m n}^{2}\left(W-X W^{2}+Y W^{3}\right)-\bar{Q} \sin \Omega t=0,
$$

in which $X=\frac{\left(a_{2}+a_{3}\right)}{-a_{1}}, Y=\frac{a_{4}}{a_{1}}, \bar{Q}=\frac{b_{4}}{I_{1}} Q$.

Seeking the solution form of Eq. (29) as $W=A \sin \Omega t$, and applying procedure like Galerkin method to Eq. (29), the frequency-amplitude relation of nonlinear forced vibration is obtained as

$$
\eta^{2}-\frac{4 \varepsilon}{\pi \omega_{m n}} \eta=\left(1-\frac{8 X}{3 \pi} A+\frac{3 Y}{4} A^{2}\right)-\frac{\bar{Q}}{A \omega_{m n}^{2}},
$$

where $\eta=\Omega / \omega_{m n}$ is the non-dimensional frequency parameter.

\subsection{Nonlinear vibration analysis of the shells under external pressure and thermal load}

In this section, we consider an imperfect FGM sandwich doubly curved shallow shell reinforced by FGM stiffeners with all immovable edges (Case 2) and exposed to a thermal environment uniformly rised from initial value $T_{i}$ to final one $T_{f}$ and $\Delta T=$ $T_{f}-T_{i}$ is independent to thickness variable $z$. The condition on immovable at all edges is fulfilled on the average sense as [7-9]

$$
\int_{0}^{b} \int_{0}^{a} \frac{\partial u}{\partial x} d x d y=0, \quad \int_{0}^{a} \int_{0}^{b} \frac{\partial v}{\partial y} d y d x=0 .
$$

Solving Eq. (31), we get $N_{x 0}, N_{y 0}$, then substituting these expressions into Eqs. (20)(22) gives

$$
\begin{aligned}
& b_{11} W+b_{12} \Psi_{x}+b_{13} \Psi_{y}+b_{14} \Psi_{x}(W+\xi h)+b_{15} \Psi_{y}(W+\xi h) \\
& +c_{1} W(W+\xi h)+c_{2} W(W+2 \xi h)+c_{3} W(W+\xi h)(W+2 \xi h)+b_{4} q \\
& +c_{4}(W+\xi h) \Delta T+c_{5} \Delta T=I_{1} \ddot{W}-2 \varepsilon I_{1} \dot{W} \\
& a_{21} W+a_{22} \Psi_{x}+a_{23} \Psi_{y}+b_{5} W(W+2 \xi h)=\bar{I}_{3} \ddot{\Phi}_{x}, \\
& a_{31} W+a_{32} \Psi_{x}+a_{33} \Psi_{y}+b_{6} W(W+2 \xi h)=\bar{I}_{3} \ddot{\Phi}_{y},
\end{aligned}
$$

where $b_{i j}$ and $c_{i}$ are found in Appendix VI.

By applying the fourth-order Runge-Kutta method for the system of Eqs. (32)-(34) combined with initial conditions, the nonlinear dynamic response of the sandwich shell with all immovable edges under external pressure and thermal loads is investigated. 
The natural frequencies of the perfect shell are determined from the system of Eqs. (32)-(34) as

$$
\left|\begin{array}{ccc}
b_{11}+c_{4} \Delta T+I_{1} \omega^{2} & b_{12} & b_{13} \\
a_{21} & a_{22}+\bar{I}_{3} \omega^{2} & a_{23} \\
a_{31} & a_{32} & a_{33}+\bar{I}_{3} \omega^{2}
\end{array}\right|=0
$$

If $\ddot{\Psi}_{x}$ and $\ddot{\Psi}_{y}$ are ignored, the system of Eqs. (32)-(34) become

$$
\begin{aligned}
I_{1} \ddot{W} & +2 \varepsilon I_{1} \dot{W}-d_{1} W-c_{4} \Delta T(W+\xi h)-d_{2} W(W+2 \xi h) \\
& -d_{3} W(W+\xi h)-d_{4} W(W+\xi h)(W+2 \xi h)-c_{5} \Delta T=b_{4} Q \sin \Omega t,
\end{aligned}
$$

in which $d_{i}$ are found in Appendix VII. By the same method as in the case of shell only under mechanical loads, from Eq. (36), the explicit expressions of the natural frequency and the frequency-amplitude relation of the perfect shell are received as below.

The natural frequency is given by

$$
\omega_{m n}=\sqrt{-\frac{\left(d_{1}+c_{4} \Delta T\right)}{I_{1}}}
$$

The frequency-amplitude relation of nonlinear forced vibration is obtained as

$$
\eta^{2}-\frac{4 \varepsilon}{\pi \omega_{m n}} I_{1} \eta=\left(1-\frac{8 \bar{X}}{3 \pi} A+\frac{3 \bar{Y}}{4} A^{2}\right)-\frac{\tilde{Q}}{A \omega_{m n}^{2}}-\frac{4 \bar{c}_{5} \Delta T}{A \pi \omega_{m n}^{2}},
$$

in which $\bar{X}=\frac{d_{2}+d_{3}}{-\left(d_{1}+c_{4} \Delta T\right)}, \bar{Y}=\frac{d_{4}}{\left(d_{1}+c_{4} \Delta T\right)}, \bar{c}_{5}=\frac{c_{5}}{I_{1}}, \tilde{Q}=\frac{b_{4} Q}{I_{1}}$.

\section{CONCLUSION}

Nonlinear vibration of the FGM sandwich doubly curved shallow shells reinforced by FGM stiffeners in thermal environment is investigated based on the FSDT. The material properties of shell are assumed to be temperature-dependent. Four material models with general power law distributions are considered. The explicit expressions of the force and moment resultants depending on stiffeners and temperature are established. By applying Galerkin's method, the analytical expressions for determining natural frequencies, nonlinear frequency-amplitude relation, and nonlinear dynamic response curves of sandwich shallow shells are obtained. In the next part, nonlinear dynamic responses will be numerically investigated by the fourth-order Runge-Kutta method.

\section{ACKNOWLEDGMENT}

This research is funded by Vietnam National Foundation for Science and Technology Development (NAFOSTED) under grant number 107.02-2015.11. 
Dang Thuy Dong, Dao Van Dung

\section{REFERENCES}

[1] X.-K. Xia and H.-S. Shen. Vibration of post-buckled sandwich plates with FGM face sheets in a thermal environment. Journal of Sound and Vibration, 314, (1), (2008), pp. 254-274. doi:10.1016/j.jsv.2008.01.019.

[2] S. M. R. Khalili and Y. Mohammadi. Free vibration analysis of sandwich plates with functionally graded face sheets and temperature-dependent material properties: A new approach. European Journal of Mechanics-A/Solids, 35, (2012), pp. 61-74. doi:10.1016/j.euromechsol.2012.01.003.

[3] S. Pandey and S. Pradyumna. Free vibration of functionally graded sandwich plates in thermal environment using a layerwise theory. European Journal of Mechanics-A/Solids, 51, (2015), pp. 55-66. doi:10.1016/j.euromechsol.2014.12.001.

[4] B. S. Aragh and M. H. Yas. Effect of continuously grading fiber orientation face sheets on vibration of sandwich panels with FGM core. International Journal of Mechanical Sciences, 53, (8), (2011), pp. 628-638. doi:10.1016/j.ijmecsci.2011.05.009.

[5] T. Dey and L. S. Ramachandra. Buckling and postbuckling response of sandwich panels under non-uniform mechanical edge loadings. Composites Part B: Engineering, 60, (2014), pp. 537-545. doi:10.1016/j.compositesb.2013.12.072.

[6] A. H. Sofiyev and N. Kuruoglu. Dynamic instability of three-layered cylindrical shells containing an FGM interlayer. Thin-Walled Structures, 93, (2015), pp. 10-21. doi:10.1016/j.tws.2015.03.006.

[7] D. V. Dung and D. T. Dong. Thermo-mechanical post-buckling analyses of functionally graded sandwich doubly curved shallow shells reinforced by FGM stiffeners with temperature-dependent material and stiffener properties resting on elastic foundations. In Proceedings of the Conference on Science and Structural Composite Materials Mechanics, Technology and Applications, Nha Trang, (2016). pp. 189-196.

[8] D. V. Dung and D. T. Dong. Nonlinear thermo-mechanical stability of eccentrically stiffened functionally graded material sandwich doubly curved shallow shells with general sigmoid law and power law according to third-order shear deformation theory. Applied Mathematics and Mechanics, 38, (2), (2017), pp. 191-216. doi:10.1007/s10483-017-2166-9.

[9] D. H. Bich, N. D. Duc, and T. Q. Quan. Nonlinear vibration of imperfect eccentrically stiffened functionally graded double curved shallow shells resting on elastic foundation using the first order shear deformation theory. International Journal of Mechanical Sciences, 80, (2014), pp. 16-28. doi:10.1016/j.ijmecsci.2013.12.009.

[10] N. D. Duc and T. Q. Quan. Transient responses of functionally graded double curved shallow shells with temperature-dependent material properties in thermal environment. European Journal of Mechanics-A/Solids, 47, (2014), pp. 101-123. doi:10.1016/j.euromechsol.2014.03.002. 


\section{APPENDIX I}

$$
\begin{aligned}
& H_{11}=H_{22}=\frac{E_{1}}{1-v^{2}}, H_{12}=\frac{E_{1} v}{1-v^{2}}, H_{66}=\frac{E_{1}}{2(1+v)}, G_{11}=G_{22}=\frac{E_{2}}{1-v^{2}}, G_{12}=\frac{E_{2} v}{1-v^{2}}, \\
& G_{66}=\frac{E_{2}}{2(1+v)}, L_{11}=L_{22}=\frac{E_{3}}{1-v^{2}}, L_{12}=\frac{E_{3} v}{1-v^{2}}, L_{66}=\frac{E_{3}}{2(1+v)}, \\
& H_{44}=K_{s}\left[\frac{E_{1}}{2(1+v)}+\frac{d_{x}}{s_{x}} \frac{E_{1 x}}{2(1+v)}\right], H_{55}=K_{s}\left[\frac{E_{1}}{2(1+v)}+\frac{d_{y}}{s_{y}} \frac{E_{1 y}}{2(1+v)}\right] ;
\end{aligned}
$$

$K_{S}$ is correction factor and

$$
\begin{aligned}
& \left(E_{1}, E_{2}, E_{3}\right)=\int_{-h / 2}^{h / 2}\left(1, z, z^{2}\right) E_{s h}(z) d z \text { and }\left(E_{1 i}, E_{2 i}, E_{3 i}\right)=\int_{h / 2}^{h / 2+h_{i}}\left(1, z, z^{2}\right) E_{s i}(z) d z \\
& \varphi_{1}^{s h}=\int_{-h / 2}^{h / 2} E_{s h}(z) \alpha_{s h}(z) \Delta T(z) d z, \quad \varphi_{1}^{s i}=\int_{h / 2}^{h / 2+h_{i}} E_{s i}(z) \alpha_{s i}(z) \Delta T(z) d z, \quad i=x, y .
\end{aligned}
$$

\section{APPENDIX II}

$$
\begin{aligned}
& H_{11}^{*}=\frac{1}{\Delta}\left(H_{11}+\frac{E_{1 x} d_{x}}{s_{x}}\right), H_{22}^{*}=\frac{1}{\Delta}\left(H_{22}+\frac{E_{1 y} d_{y}}{s_{y}}\right), H_{12}^{*}=\frac{H_{12}}{\Delta}, \\
& \Delta=\left(H_{11}+\frac{E_{1 x} d_{x}}{s_{x}}\right)\left(H_{22}+\frac{E_{1 y} d_{y}}{s_{y}}\right)-H_{12}^{2}, H_{66}^{*}=\frac{1}{H_{66}}, \\
& G_{11}^{*}=H_{22}^{*}\left(G_{11}+\frac{E_{2 x} d_{x}}{s_{x}}\right)-H_{12}^{*} G_{12}, G_{12}^{*}=H_{22}^{*} G_{12}-H_{12}^{*}\left(G_{22}+\frac{E_{2 y} d_{y}}{s_{y}}\right), \\
& G_{21}^{*}=H_{11}^{*} G_{12}-H_{12}^{*}\left(G_{11}+\frac{E_{2 x} d_{x}}{s_{x}}\right), \\
& G_{22}^{*}=H_{11}^{*}\left(G_{22}+\frac{E_{2 y} d_{y}}{s_{y}}\right)-H_{12}^{*} G_{12}, G_{66}^{*}=\frac{G_{66}}{H_{66}}, \\
& L_{11}^{*}=L_{11}+\frac{E_{3 x} d_{x}}{s_{x}}-G_{11}^{*}\left(G_{11}+\frac{E_{2 x} d_{x}}{s_{x}}\right)-G_{12} G_{21}^{*}, \\
& L_{12}^{*}=L_{12}-G_{12}^{*}\left(G_{11}+\frac{E_{2 x} d_{x}}{s_{x}}\right)-G_{12} G_{22}^{*}, \\
& L_{21}^{*}=L_{12}-G_{21}^{*}\left(G_{22}+\frac{E_{2 y} d_{y}}{s_{y}}\right)-G_{12} G_{11}^{*}, L_{66}^{*}=L_{66}-G_{66} G_{66}^{*} .
\end{aligned}
$$




\section{APPENDIX III}

$$
\begin{aligned}
& a_{11}=-\left(\frac{\beta^{2}}{R_{x}}+\frac{\alpha^{2}}{R_{y}}\right)^{2} \frac{1}{M}-K_{1}-K_{2}\left(\alpha^{2}+\beta^{2}\right)-\left(H_{44} \alpha^{2}+H_{55} \beta^{2}\right) \text {, } \\
& a_{12}=-H_{44} \alpha+\left(\frac{\beta^{2}}{R_{x}}+\frac{\alpha^{2}}{R_{y}}\right)\left[\left(G_{66}^{*}-G_{11}^{*}\right) \alpha \beta^{2}-G_{21}^{*} \alpha^{3}\right] \frac{1}{M^{\prime}}, \\
& a_{13}=-H_{55} \beta+\left(\frac{\beta^{2}}{R_{x}}+\frac{\alpha^{2}}{R_{y}}\right)\left[\left(G_{66}^{*}-G_{22}^{*}\right) \alpha^{2} \beta-G_{12}^{*} \beta^{3}\right] \frac{1}{M^{\prime}} \\
& a_{14}=-\frac{8 \alpha^{2} \beta^{2} \delta_{m} \delta_{n}\left[\left(G_{66}^{*}-G_{11}^{*}\right) \alpha \beta^{2}-G_{21}^{*} \alpha^{3}\right]}{3 m n \pi^{2} M} \text {, } \\
& a_{15}=-\frac{8 \alpha^{2} \beta^{2} \delta_{m} \delta_{n}\left[\left(G_{66}^{*}-G_{22}^{*}\right) \alpha^{2} \beta-G_{12}^{*} \beta^{3}\right]}{3 m n \pi^{2} M}, \\
& b_{1}=\frac{8 \alpha^{2} \beta^{2} \delta_{m} \delta_{n}}{3 m n \pi^{2} M}\left(\frac{\beta^{2}}{R_{x}}+\frac{\alpha^{2}}{R_{y}}\right), \quad b_{2}=\frac{\delta_{m} \delta_{n}}{6 m n \pi^{2}}\left(\frac{\alpha^{2}}{H_{22}^{*} R_{x}}+\frac{\beta^{2}}{H_{11}^{*} R_{y}}\right), \\
& b_{3}=-\frac{1}{16}\left(\frac{\beta^{4}}{H_{11}^{*}}+\frac{\alpha^{4}}{H_{22}^{*}}\right) \text {, } \\
& a_{21}=\left(\frac{\beta^{2}}{R_{x}}+\frac{\alpha^{2}}{R_{y}}\right)\left[G_{21}^{*} \alpha^{3}+\left(G_{11}^{*}-G_{66}^{*}\right) \alpha \beta^{2}\right] \frac{1}{M}+H_{44} \alpha, \\
& a_{22}=L_{11}^{*} \alpha^{2}+L_{66}^{*} \beta^{2}+H_{44}+\left[G_{21}^{*} \alpha^{3}+\left(G_{11}^{*}-G_{66}^{*}\right) \alpha \beta^{2}\right]^{2} \frac{1}{M^{\prime}} \\
& a_{31}=\left(\frac{\beta^{2}}{R_{x}}+\frac{\alpha^{2}}{R_{y}}\right)\left[G_{12}^{*} \beta^{3}+\left(G_{22}^{*}-G_{66}^{*}\right) \alpha^{2} \beta\right] \frac{1}{M}+H_{55} \beta, \\
& a_{23}=\left(L_{12}^{*}+L_{66}^{*}\right) \alpha \beta+\left[G_{12}^{*} \beta^{3}+\left(G_{22}^{*}-G_{66}^{*}\right) \alpha^{2} \beta\right]\left[G_{21}^{*} \alpha^{3}+\left(G_{11}^{*}-G_{66}^{*}\right) \alpha \beta^{2}\right] \frac{1}{M} \text {, } \\
& b_{4}=\frac{4 \delta_{m} \delta_{n}}{m n \pi^{2}}, \quad b_{5}=-\frac{G_{21}^{*}}{H_{11}^{*}} \frac{2 n \pi}{3 a b^{2}} \delta_{m} \delta_{n} \\
& a_{32}=\left(L_{21}^{*}+L_{66}^{*}\right) \alpha \beta+\left[G_{21}^{*} \alpha^{3}+\left(G_{11}^{*}-G_{66}^{*}\right) \alpha \beta^{2}\right]\left[G_{12}^{*} \beta^{3}+\left(G_{22}^{*}-G_{66}^{*}\right) \alpha^{2} \beta\right] \frac{1}{M^{\prime}} \\
& b_{6}=-\frac{G_{12}^{*}}{H_{22}^{*}} \frac{2 m \pi}{3 a^{2} b} \delta_{m} \delta_{n} \\
& a_{33}=L_{22}^{*} \beta^{2}+L_{66}^{*} \alpha^{2}+H_{55}+\left[G_{12}^{*} \beta^{3}+\left(G_{22}^{*}-G_{66}^{*}\right) \alpha^{2} \beta\right]^{2} \frac{1}{M^{\prime}},
\end{aligned}
$$

where

$$
M=H_{11}^{*} \alpha^{4}+H_{22}^{*} \beta^{4}+\left(H_{66}^{*}-2 H_{12}^{*}\right) \alpha^{2} \beta^{2} .
$$




\section{APPENDIX IV}

$$
\begin{aligned}
& a_{1}=a_{11}+a_{12} \frac{a_{23} a_{31}-a_{33} a_{21}}{a_{22} a_{33}-a_{23} a_{32}}+a_{13} \frac{a_{32} a_{21}-a_{22} a_{31}}{a_{22} a_{33}-a_{23} a_{32}}, \\
& a_{2}=a_{12} \frac{a_{23} b_{6}-a_{33} b_{5}}{a_{22} a_{33}-a_{23} a_{32}}+a_{13} \frac{a_{32} b_{5}-a_{22} b_{6}}{a_{22} a_{33}-a_{23} a_{32}}+b_{2}, \\
& a_{3}=a_{14} \frac{a_{23} a_{31}-a_{33} a_{21}}{a_{22} a_{33}-a_{23} a_{32}}+a_{15} \frac{a_{32} a_{21}-a_{22} a_{31}}{a_{22} a_{33}-a_{23} a_{32}}+b_{1}, \\
& a_{4}=a_{14} \frac{a_{23} b_{6}-a_{33} b_{5}}{a_{22} a_{33}-a_{23} a_{32}}+a_{15} \frac{a_{32} b_{5}-a_{22} b_{6}}{a_{22} a_{33}-a_{23} a_{32}}+b_{3},
\end{aligned}
$$

\section{APPENDIX V}

$$
\begin{aligned}
& t_{11}=\frac{\delta_{m} \delta_{n}}{m n \pi^{2}}\left[G_{5}\left(H_{22}^{*} \beta^{2}-H_{12}^{*} \alpha^{2}\right)-\frac{1}{R_{x}}\right], t_{12}=\frac{\delta_{m} \delta_{n}}{m n \pi^{2}}\left[G_{3}\left(H_{22}^{*} \beta^{2}-H_{12}^{*} \alpha^{2}\right)-G_{11}^{*} \alpha\right], \\
& t_{14}=\frac{1}{8} \alpha^{2}, \quad t_{24}=\frac{1}{8} \beta^{2}, \quad t_{13}=\frac{\delta_{m} \delta_{n}}{m n \pi^{2}}\left[G_{4}\left(H_{22}^{*} \beta^{2}-H_{12}^{*} \alpha^{2}\right)-G_{12}^{*} \beta\right], \\
& t_{21}=\frac{\delta_{m} \delta_{n}}{m n \pi^{2}}\left[G_{5}\left(H_{11}^{*} \alpha^{2}-H_{12}^{*} \beta^{2}\right)-\frac{1}{R_{y}}\right], t_{22}=\frac{\delta_{m} \delta_{n}}{m n \pi^{2}}\left[G_{3}\left(H_{11}^{*} \alpha^{2}-H_{12}^{*} \beta^{2}\right)-G_{21}^{*} \alpha\right], \\
& t_{23}=\frac{\delta_{m} \delta_{n}}{m n \pi^{2}}\left[G_{4}\left(H_{11}^{*} \alpha^{2}-H_{12}^{*} \beta^{2}\right)-G_{22}^{*} \beta\right],
\end{aligned}
$$

in which

$$
\begin{aligned}
& G_{3}=-\left[\left(G_{66}^{*}-G_{11}^{*}\right) \alpha \beta^{2}-G_{21}^{*} \alpha^{3}\right] \frac{1}{M_{1}}, \\
& G_{4}=-\left[\left(G_{66}^{*}-G_{22}^{*}\right) \alpha^{2} \beta-G_{12}^{*} \beta^{3}\right] \frac{1}{M_{1}}, \\
& G_{5}=\left(\frac{\beta^{2}}{R_{x}}+\frac{\alpha^{2}}{R_{y}}\right) \frac{1}{M_{1}},
\end{aligned}
$$

where $M_{1}=H_{11}^{*} \alpha^{4}+\left(H_{66}^{*}-2 H_{12}^{*}\right) \alpha^{2} \beta^{2}+H_{22}^{*} \beta^{4}$.

\section{APPENDIX VI}

$$
\begin{aligned}
& b_{11}=a_{11}+\frac{b_{4}}{R_{x}} \frac{H_{11}^{*} t_{11}+H_{12}^{*} t_{21}}{D}+\frac{b_{4}}{R_{y}} \frac{H_{22}^{*} t_{21}+H_{12}^{*} t_{11}}{D}, \\
& b_{12}=a_{12}+\frac{b_{4}}{R_{x}} \frac{H_{11}^{*} t_{12}+H_{12}^{*} t_{22}}{D}+\frac{b_{4}}{R_{y}} \frac{H_{11}^{*} t_{22}+H_{12}^{*} t_{12}}{D}, \\
& b_{13}=a_{13}++\frac{b_{4}}{R_{x}} \frac{H_{11}^{*} t_{13}+H_{12}^{*} t_{23}}{D}+\frac{b_{4}}{R_{y}} \frac{H_{22}^{*} t_{23}+H_{12}^{*} t_{13}}{D},
\end{aligned}
$$




$$
\begin{aligned}
& b_{14}=a_{14}-\alpha^{2} \frac{H_{11}^{*} t_{12}+H_{12}^{*} t_{22}}{D}-\beta^{2} \frac{H_{22}^{*} t_{22}+H_{12}^{*} t_{12}}{D}, \\
& b_{15}=a_{15}-\alpha^{2} \frac{H_{11}^{*} t_{13}+H_{12}^{*} t_{23}}{D}-\beta^{2} \frac{H_{22}^{*} t_{23}+H_{12}^{*} t_{13}}{D}, \\
& c_{1}=b_{1}-\alpha^{2} \frac{H_{11}^{*} t_{11}+H_{12}^{*} t_{21}}{D}-\beta^{2} \frac{H_{22}^{*} t_{21}+H_{12}^{*} t_{11}}{D}, \\
& c_{2}=b_{2}+\frac{b_{4}}{R_{x}} \frac{H_{11}^{*} t_{14}+H_{12}^{*} t_{24}}{D}+\frac{b_{4}}{R_{y}} \frac{H_{22}^{*} t_{24}+H_{12}^{*} t_{14}}{D}, \\
& c_{3}=b_{3}-\alpha^{2} \frac{H_{11}^{*} t_{14}+H_{12}^{*} t_{24}}{D}-\beta^{2} \frac{H_{22}^{*} t_{24}+H_{12}^{*} t_{14}}{D}, \\
& c_{4}=\frac{\alpha^{2}+\beta^{2}}{1-v} P_{1}+\frac{d_{x}}{s_{x}} \alpha^{2} P_{2}+\frac{d_{y}}{s_{y}} \beta^{2} P_{3}, \\
& c_{5}=-\frac{1}{1-v}\left(\frac{1}{R_{x}}+\frac{1}{R_{y}}\right) b_{4} P_{1}-\frac{d_{x}}{s_{x} R_{x}} b_{4} P_{2}-\frac{d_{y}}{s_{y} R_{y}} b_{4} P_{3},
\end{aligned}
$$

in which $D=H_{11}^{*} H_{22}^{*}-H_{12}^{* 2}$ and

$$
P_{1}=\int_{-h / 2}^{h / 2} E_{s h}(z) \alpha_{s h}(z) d z, \quad P_{2}=\int_{h / 2}^{h / 2+h_{x}} E_{s x}(z) \alpha_{s x}(z) d z, \quad P_{3}=\int_{h / 2}^{h / 2+h_{y}} E_{s y}(z) \alpha_{s y}(z) d z .
$$

\section{APPENDIX VII}

$$
\begin{aligned}
& d_{1}=b_{11}+b_{12} \frac{a_{23} a_{31}-a_{33} a_{21}}{a_{22} a_{33}-a_{23} a_{32}}+b_{13} \frac{a_{32} a_{21}-a_{22} a_{31}}{a_{22} a_{33}-a_{23} a_{32}}, \\
& d_{2}=b_{12} \frac{a_{23} b_{6}-a_{33} b_{5}}{a_{22} a_{33}-a_{23} a_{32}}+b_{13} \frac{a_{32} b_{5}-a_{22} b_{6}}{a_{22} a_{33}-a_{23} a_{32}}+c_{2}, \\
& d_{3}=b_{14} \frac{a_{23} a_{31}-a_{33} a_{21}}{a_{22} a_{33}-a_{23} a_{32}}+b_{15} \frac{a_{32} a_{21}-a_{22} a_{31}}{a_{22} a_{33}-a_{23} a_{32}}+c_{1}, \\
& d_{4}=b_{14} \frac{a_{23} b_{6}-a_{33} b_{5}}{a_{22} a_{33}-a_{23} a_{32}}+b_{15} \frac{a_{32} b_{5}-a_{22} b_{6}}{a_{22} a_{33}-a_{23} a_{32}}+c_{3} .
\end{aligned}
$$

